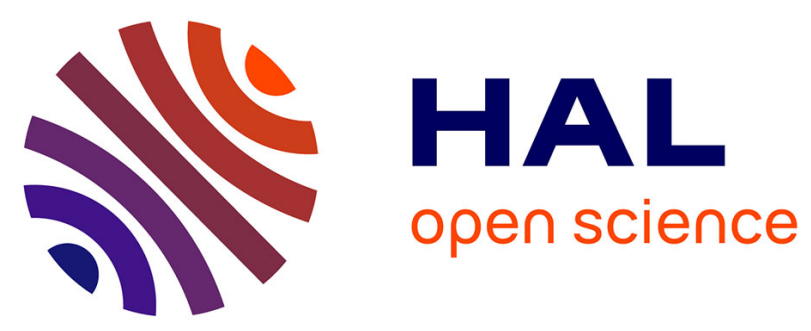

\title{
Performance analysis of Ethernet Powerlink protocol: Application to a new lift system generation
}

\author{
Ayoub Soury, Melek Charfi, Denis Genon-Catalot, Jean-Marc Thiriet
}

\section{To cite this version:}

Ayoub Soury, Melek Charfi, Denis Genon-Catalot, Jean-Marc Thiriet. Performance analysis of Ethernet Powerlink protocol: Application to a new lift system generation. ETFA 2015 - 20th IEEE International Conference on Emerging Technologies and Factory Automation, IEEE, Sep 2015, Luxembourg, Luxembourg. 6 p., 10.1109/ETFA.2015.7301492 . hal-01233841

\section{HAL Id: hal-01233841 \\ https://hal.univ-grenoble-alpes.fr/hal-01233841}

Submitted on 2 Dec 2015

HAL is a multi-disciplinary open access archive for the deposit and dissemination of scientific research documents, whether they are published or not. The documents may come from teaching and research institutions in France or abroad, or from public or private research centers.
L'archive ouverte pluridisciplinaire HAL, est destinée au dépôt et à la diffusion de documents scientifiques de niveau recherche, publiés ou non, émanant des établissements d'enseignement et de recherche français ou étrangers, des laboratoires publics ou privés. 


\title{
Performance analysis of Ethernet Powerlink protocol: Application to a new lift system generation
}

\author{
Ayoub Soury ${ }^{* \dagger}$, Melek Charfi ${ }^{\dagger}$, Denis Genon-Catalot ${ }^{\dagger}$ and Jean-Marc Thiriet ${ }^{* \ddagger}$ \\ ${ }^{*}$ Univ. Grenoble Alpes, Gipsa-lab, F-38000 Grenoble, France \\ ${ }^{\dagger}$ Univ. Grenoble Alpes, LCIS, F-26000 Valence, France \\ ${ }^{\ddagger}$ CNRS, Gipsa-lab, F-38000 Grenoble, France \\ Email: \{ayoub.soury, denis.genon-catalot\}@1cis.grenoble-inp.fr; jean-marc.thiriet@gipsa-lab.grenoble-inp.fr
}

\begin{abstract}
To ensure control, present lifts use the Controller Area Network (CAN) bus for transmitting commands between components. Although it is largely adopted in the industrial process, CAN is not able to guarantee a sufficient throughput to transmit multimedia data or to meet the requirements of some safety standards. In this paper, we present a transition case from electrical/electromechanical components to a networked control system. The main element we focus on in the lift system is the safety chain. We propose to build the lift communication system around real-time Ethernet for more efficiency, smartness and safety. Furthermore, the use of the openSAFETY protocol as a safety layer over the real-time Ethernet allows the achievement of the required Safety Integrity Level (SIL). This adopted solution should meet the adopted standard IEC 61508 requirements.
\end{abstract}

\section{INTRODUCTION}

Nowadays, Ethernet is commonly used for the home and office environment. Ethernet allows connecting any type of device due to its fast and easy installation, and interoperability. In addition, Ethernet has a large potential to become an ideal solution for automation technology. However, it is known that classic Ethernet is not suitable for industrial networking because of the Carrier Sense Multiple Access with Collision Detection (CSMA-CD) mechanism. Modern industrial automation systems consist of multiple devices exchanging information through communication networks. Traditionally, these local networks are restrained to an industrial plant, but recently the trend is to interconnect them remotely through Internet. This allows the collect of available supervisory and control data to apply maintenance and diagnosis operations on the running devices [1]. In [2], authors explain that Industrial Control System (ICS) is a general term that refers to a set of interconnected systems that include Programmable Logic Controllers (PLC), Distributed Control Systems (DCS) and Supervisory Control and Data Acquisition (SCADA). They allow automation and control of industrial processes. They include industrial processes for Power Generation, Gas Transportation, Aero-Space Industry, Food Industry, Automotive, etc.. The adoption of Ethernet technology cannot be accepted if the field area loses its principal features:

- Time-deterministic communication;

- Time-synchronized actions between field devices;
- Efficient and frequent exchange of very small data records.

Industrial Ethernet must fully retain the office Ethernet communication capability. Using Ethernet for industrial communication brings some advantages; Solutions, that uses unified physical layer leading to lower prices and vast choice of network components. In this paper, we detail the development of an experimental test bed dedicated to industrial systems (lift control system). The test bed is built on real hardware (STM32 cards). We designed and implemented a networked process control system using an Ethernet-based protocol in their communication layer: the Powerlink + OpenSAFETY. Using the developed experimental process, we analyze Ethernet Powerlink protocol (EPL) and its safety extension (OpenSAFETY) to reach a minimum cycle time in industrial networked architecture around a real-time operating system. This solution allows to meet a safety standard requirements for the lift system. The remainder of the paper is organized as follows. In section II, we present the state of the art for deterministic industrial communication based on Ethernet. After the state of art, we justify our choice for the Real-Time Ethernet (RTE) protocol. Then, we detail the classification of RTE. In section III, we describe the adopted protocol for our project and we compare its model with the model of a classical communication (OSI model). Our adopted approach and results are presented in section IV and V. In fact, section IV shows our architecture for the lift control system. In this section we explain how we do integrate RTE in our networked industrial system and we will present the theoretical analysis and experimental measures. In Section V, we focus on new concept in the lift control system: the safety over RTE in lift control system, so we will interpret the obtained results and discussing it. Finally, in Section VI we make our conclusions.

\section{Classes of Real-time Ethernet}

In modern lift architectures, sensors and actuators are connected through the CAN bus [3]. The integration and system management are declared in the CANopen framework. The CAN in Automation group (CiA) adjusts and refines the CANopen dictionary to have a new suitable CANopen 
called CANopen-Lift [3]. This kind of frameworks gives the designer full variables access in the lift control system. For the future lift management in buildings, it is imperative to ensure data video, multimedia support, etc.. Despite the growth up to $10 \mathrm{Mbits} / \mathrm{s}$ of the last draft of CAN, stakeholders aim for a global solution supporting IP connection for web services, remote maintenance, etc.. Stakeholders are looking for a unique interface for the control and services. In our contribution we make a survey of the real time Ethernet and reuse the CANopen communication in the head of IP networks. We can classify different approaches of Real-Time Ethernet (RTE) realization into 3 classes [4] as shown in Fig.1. All RTE approaches use identical physical layers. Non real-time applications use of the Ethernet protocols as defined in ISO 8802-3 and the TCP/UDP/IP protocol suite.

Class 1: Realization on top of TCP/IP:

The TCP/UDP/IP protocol stack is used here without any modification [5]. To achieve a cycle time equal to $100 \mathrm{~ms}$, the protocol stack needs reasonable resources in processing power and memory which introduces non-deterministic delays in the communication [3].

- Class 1 protocols: ModBus/TCP [6][7], Ethernet/IP [8][9], etc..

\section{Class 2: Realization on top of Ethernet:}

These realizations are required by PLCs and they do not change the Ethernet communication principle (i.e. the data link layer). Their own protocol stack replaces TCP/UDP/IP protocol stack to obtain a cycle time $<10 \mathrm{~ms}$.

- Class 2 protocols: Profinet RT [10][11], Ethernet Powerlink [12][13], EtherCAT class B [14], etc..

\section{Class 3: Realization with modified Ethernet:}

The data link layer is modified within this approach to achieve a cycle time $<1 \mathrm{~ms}$. This class offers a high synchronization

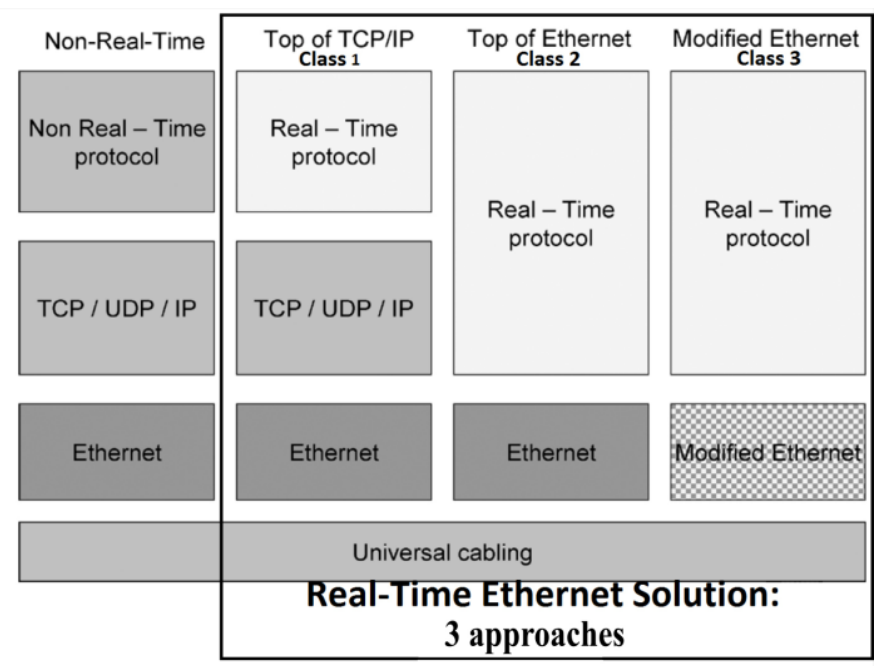

Fig. 1. Classification of industrial Ethernet protocols. precision. These realizations are required by Motion Control. They implement specific hardware to reduce the classic stack.

- Class 3 protocols: Profinet IRT [5] [10], SERCOS [15], EtherCAT class A [5][14][16], TTEthernet [17][18][19], etc..

\section{ETHERNET POWERLINK PROTOCOL}

Ethernet Powerlink (EPL) was originally developed to transfer the CANopen framework over IP stack. Some german companies like B\&R GmbH support the EPL developer for industrial control application on real time Ethernet. Supported by Ethernet Powerlink Standardization Group (EPSG), this protocol was certified ISO in 2004 and IEC 61784 (Communication Profile Families CPF-13) [12]. This standard, managed by the EPSG, is still opened in order to be specified for industrial control [20].

\section{A. Model of Ethernet Powerlink}

EPL is considered as a class 2 protocol i.e over a Mac 802.3 standard (with $88 A B$ Ethetype value). It could achieve a cycle time less than $10 \mathrm{~ms}$. As shown in Fig. ??, EPL network uses standard Ethernet interface (all Ethernet usual port will support EPL network). The Data Link Layer (EPL DLL) is specified over the Ethernet Medium Access Control (MAC). The application layer offers the process data support with an Object Dictionary (OD) inherited from the CANopen stack. Regarding IEEE 802.3 Protocol Data Unit (PDU), EPL add five additional fields allocated in the three bytes of the Ethernet data field. The "MessageType" field is used to identify the different exchanges in an EPL network. It is used in our approach to calculate the cycle time in our industrial network (used in sniffing with wireshark). At least, with the EPL, Ethernet protocol was not hardware modified. Therefore a standard communication over TCP/IP is still possible.

\section{B. Slot Communication network Management}

Powerlink Ethernet is based on the principle of using a master-slave scheduling system on a shared Ethernet segment called Slot Communication Network Management (SCNM) [21]. EPL defines two types of stations: Managing Node $(\mathrm{MN})$ and Controlled Node (CNs). The master (MN which is unique) ensures the real-time access to the cyclic data and lets standard TCP/IP frame pass through, only in specific timeslots. The unique MN in the network is responsible for traffic scheduling and executes regular polls of several CNs (up to 240). It allocates time slot of data transmission for each node in a cyclic manner within a guaranteed cycle. The masterslave relationship is established by means of a continuously repeated sequence of operations namely cycle shown in Fig. 2. Generally, we can split the EPL cycle into 4 sections as shown in Fig. 2. Effectively, the cycle is composed of two different periods: the Isochronous and the Asynchronous period. At the beginning of each cycle, the unique $\mathrm{MN}$ in the network broadcasts a "Start of Cycle" ( $\mathrm{SoC}$ ) frame to synchronize all the CNs. Then, the isochronous period is started. During this period, the MN polls each $\mathrm{CN}$ in sequence by means of "Poll 
Request" (PReq) frame to transfer output data. When a CN gets the PReq frame, it should broadcast "Poll Response" (PRes) frame to the network to transfer input data. Then every node (MN or $\mathrm{CN}$ ) can detect this PRes frame. At the end of an isochronous period, the MN broadcasts the PRes frame to all CNs. Only the MN can start and finish the isochronous period. The MN broadcasts the "Start of Asynchronous" ( $\mathrm{SoA}$ ) frame to notify the beginning of the acyclic period to all the $\mathrm{CNs}$. Via the SoA frame, the MN grants a unique $\mathrm{CN}$ to send an acyclic message. Then, the designed $\mathrm{CN}$ uses the "Asynchronous Send" (ASnd) frame to transmit its acyclic data. When $\mathrm{CN}$ finishes sending its acyclic message, an idle phase starts. The MN waits for the remaining time before starting a new EPL cycle. The SCNM mechanism ensures that only one network device can access to the network and there are no collisions, thus providing deterministic communication and real-time network transmission. The CNs may be accessed every cycle or every $\mathrm{n}^{\text {th }}$ cycle. It shall send back a PRes frame to $\mathrm{MN}$. The duration of the asynchronous phase may vary from one cycle to the next. If a $\mathrm{CN}$ is assigned to send and there is no information about the length of the expected asynchronous frame available at the MN, the next reduced Powerlink cycle shall not start until at least the timeout given by the length of a maximum size Ethernet frame.

\section{PRINCIPLE AND REALIZATION: SAFETY CHAIN IN LIFT SYSTEM}

\section{A. Adopted approach: New lift safety architecture}

In this part, we describe our target networked industrial system. As shown in Fig. 3, the lift system is composed essentially of a car, a safety chain, a controller and a motor [22]. To move the lift car, the controller checks that all contacts of the safety chain are closed and then controls the motor. Communication between components is ensured through industrial protocols (fieldbus). The safety chain is composed of serial contacts range that controls the power of a dedicated Input. The displacement of the car is possible only if all contacts are closed. Currently, this is the solution adopted by all lift constructors. So, making the safety chain smarter is a business need. To facilitate the control and automation of the safety chain lift, we proposed a new approach using

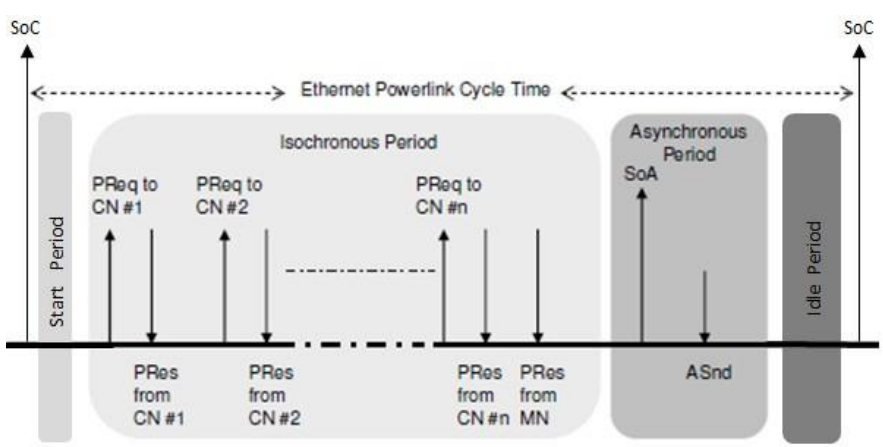

Fig. 2. Ethernet Powerlink cycle. an industrial network to replace the contact in the existing safety chain with smarter devices identifiable and controlled by a programmable logic controller (PLC). To improve the

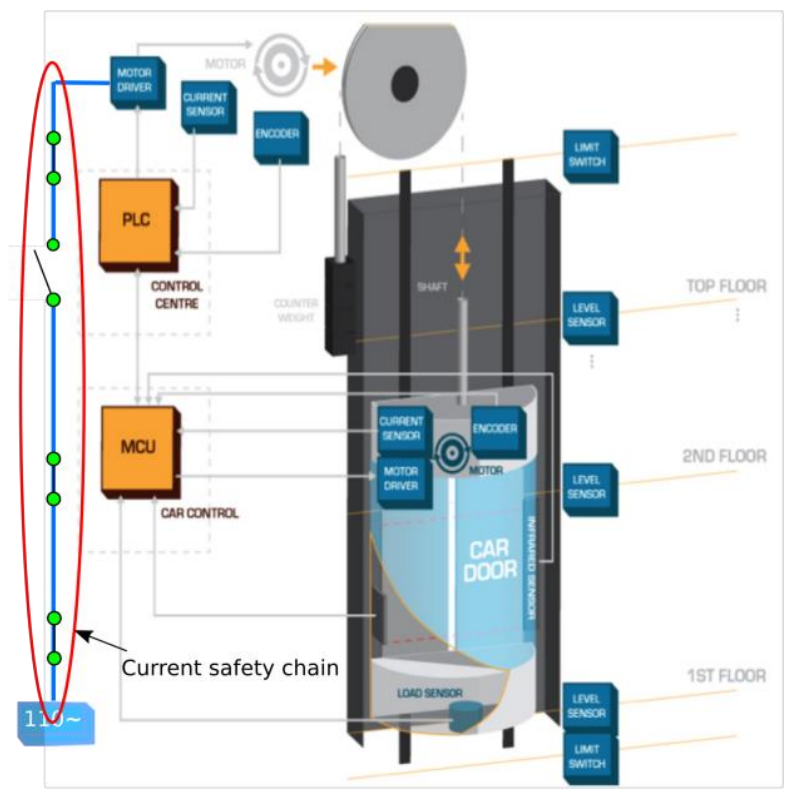

Fig. 3. Current safety chain in the lift architecture.

components behavior we used a real-time operating system in this smart device to achieve the temporal requirement as shown in Fig. 4. Respecting Fig. 4, the lift control system becomes able to collect information from the safety chain by means of slaves, make the decision to move the car, control the PLC from the master and give orders to the motor. The adopted architecture must model the communication in the safety chain. The automation of the safety chain has to ensure a communication through a protocol based on Ethernet and deterministic real time among the different nodes of the network. The following networked safety chain uses the Ethernet Powerlink as communication protocol. This Powerlink network, consisted of one $\mathrm{MN}$ and two CNs is established for the purpose of explaining the realization process of safety chain. In Fig. 6, as soon as MN starts the EPL communications by broadcasting the $S o C$ frame to all nodes in the network.

\section{B. Configuration of parameters of protocols stack}

Ethernet Powerlink introduces object-oriented communication. Indeed, in application Layer, EPL uses the Objects Dictionary (OD). It defines all the objects that can be exchanged in the network. The OD may contain a maximum of 65536 $\left(2^{16}\right)$ inputs which are addressed through a 16-bit index. The communication profile area at indices 1000 through 1FFF contains the communication specific parameters for the Powerlink network. These inputs are common to all devices. The setting of configuration parameters of protocols stack is the key to enable the network to transmit information in accordance with the user agreement. In addition, the configuration parameters are stored in object index of OD. For instance, to control the timing behavior of the Powerlink network traffic, we store our 
configuration in object index 1006. This object defines the communication cycle time interval in $\mu \mathrm{s}$. The $1 \mathrm{~F} 9 \mathrm{C}$ object assigns nodes to a particular isochronous slot, and the 1F8B object defines the PReq payload data size in octet for each configured node. The 1F8D object configures the PRes payload data. There are two types of Process Data Object (PDO) for the critical data transfer in real time. There is the Transmitted Process Data Objects (TPDO) which is configured at the sending node and stored in object index from 1800 to $1 \mathrm{AFF}$. While, in the receiver node, there is the Received Process Data Objects (RPDO) stored in object index from 1400 to $16 \mathrm{FF}$. To reduce the cycle time, users need to pay much more attention to the definition of the following object indexes (payload size, frame timeout, etc.).

\section{Theoretical analysis and experimental measurements}

In order to determine the optimized cycle time of our Powerlink network, we shall choose the appropriate network variables configuration. We have made some measurements on the cycle time in our EPL network with two slaves. The results are inserted in Table I.

TABLE I

Results of Powerlink Proto col tests for two Slaves (ms)

\begin{tabular}{ccccc} 
Soc $\rightarrow$ Peq & PReq $\rightarrow$ PRes & Soc $\rightarrow$ SoA & SoA $\rightarrow$ ASnd & Cycle time \\
\hline 0.1 & 10 & 50 & 30 & 100 \\
0.1 & 08 & 40 & 20 & 80 \\
0.1 & 05 & 25 & 15 & 50 \\
0.1 & 03 & 15 & 10 & 30 \\
0.1 & 01 & 05 & 03 & 10 \\
\hline
\end{tabular}

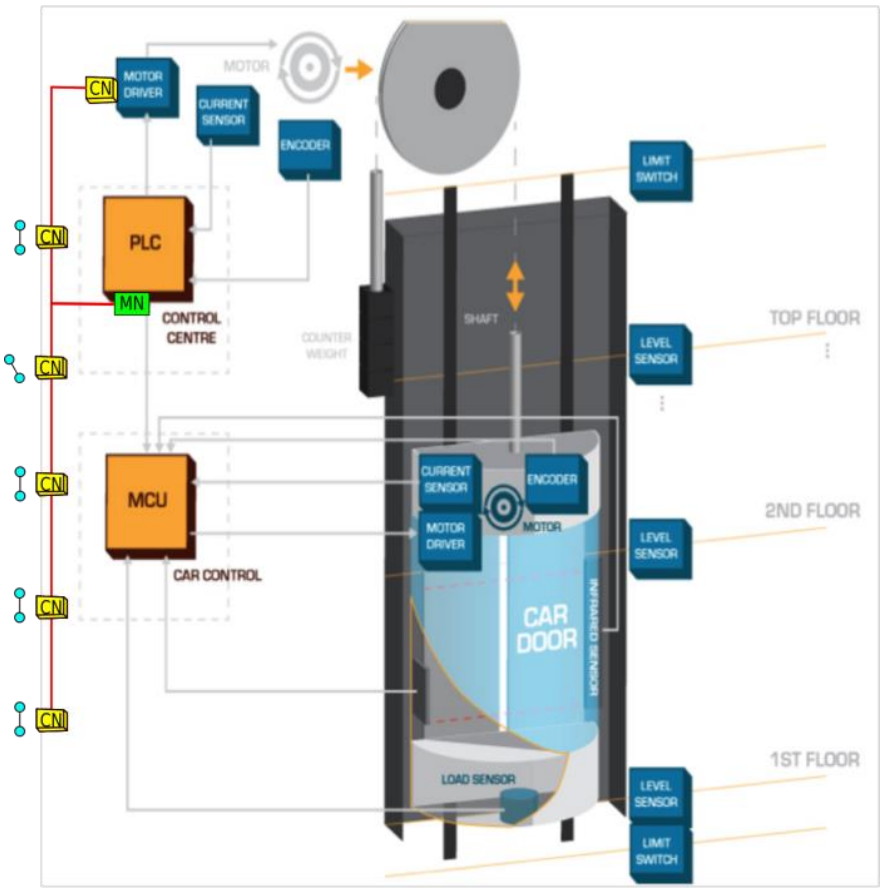

Fig. 4. Modified safety chain in lift control system.

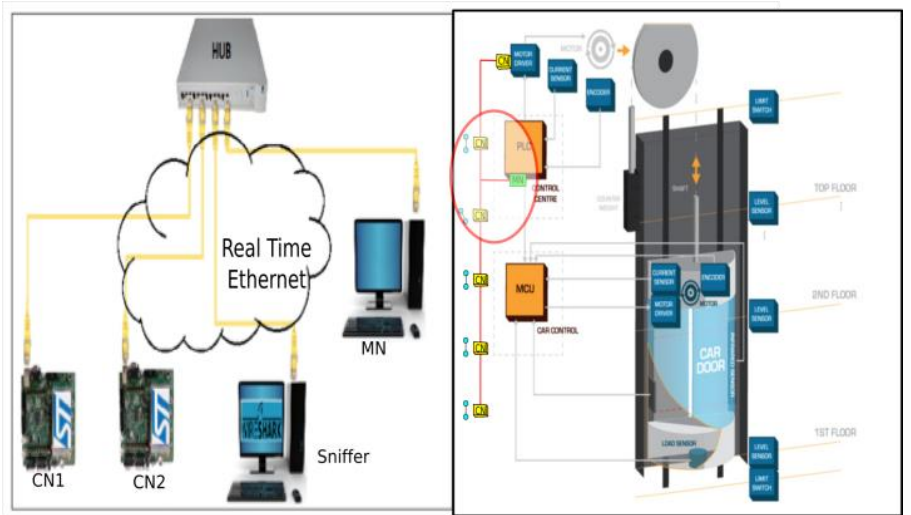

Fig. 5. Realization process of safety chain.

- Soc $\rightarrow \mathrm{P}_{\mathrm{Req}}$ : The time between the SoC frame and the first frame PReq.

- $\mathrm{P}_{\mathrm{Req}} \rightarrow \mathrm{P}_{\mathrm{Res}}$ : The maximum time required by $\mathrm{CN}$ to reply to PReq frame.

- Soc $\rightarrow$ SoA: The maximum time of the isochronous period.

- $S o A \rightarrow$ Asnd The maximum time required by the $\mathrm{CN}$ to issue ASnd frame.

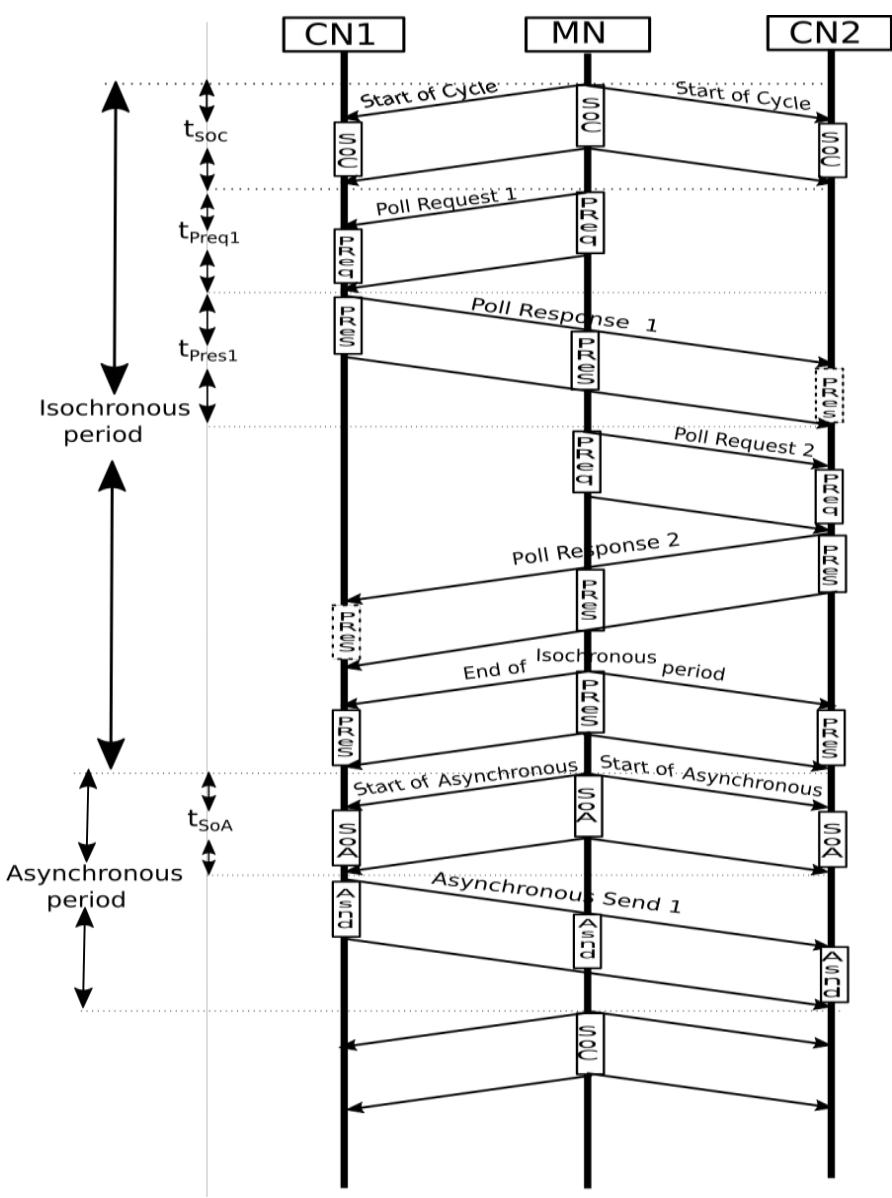

Fig. 6. Sequence Diagram of EPL Communication. 
In EPL network containing n CNs, we can identify in each cycle the following messages:

- $1 S o C$ frame sized 64 bytes for all network.

- n PReq frames (for each CN one PReq frame). Each frame sized 64 (minimum)-1518 (maximum) bytes.

- $\mathrm{n}$ PRes frames (from each CN). Each frame sized 64-1518 bytes.

- 1 PRes frame (broadcasting by MN to declare the isochronous period end). It sized $64-1518$ bytes

- $1 S o A$ frame sized 64 bytes for all network.

- 1 ASnd frame sized 318-1518 bytes (at least).

The theoretical cycle time depends on the size of the messages and the number of slaves in the network. The formula computation of the minimum cycle time is as follows (1)

Minimum cycle $=\frac{((2 n+3) \times 64+318) \times 8}{\text { Real rate }}$
$+2 \times(n+2) \times$ IF G + Jitter start

n: Slave number,

IFG: Inter Frame Gap (default value $=960 \mathrm{n}$ s)

Jitter start: $20 \mathrm{n} \mathrm{s}$

Fast Ethernet has a theoretical speed of $100 \mathrm{Mbits} / \mathrm{sec}$. In our case (with only two slaves and one master, e.g $n=2$ ), we have to reach $100 \mu \mathrm{s}$.

Based on the diagram of sequences shown in Fig. 6, we can deduce in another way the formula of the cycle time in the EPL network:

$$
\text { Cycle time }=\mathrm{t}_{\mathrm{SoC}}+\underset{\substack{\mathrm{k}=1 \\+\mathrm{t}_{\mathrm{SoA}}+\mathrm{t}_{\mathrm{ASnd}}}}{\left.\mathbf{P}_{\mathrm{Preq}}+\mathrm{t}_{\text {Pres }_{\mathrm{k}}}\right)+\mathrm{t}_{\text {Pres }}}
$$

Theoretically, a PowerLink cycle may reach values less than $1 \mathrm{~ms}$ (around $400 \mu \mathrm{s}$ [4]). But in theory, theory and practice are the same. In practice, they are not. These values $(400 \mu \mathrm{s})$ can be achieved by using a specific hardware in the network components (MN or $\mathrm{CN}$ ) as FPGA (Field Programmable Gate Array) or ASIC (Application Specific Integrated circuit) allowing very fast data processing.

In our implementation, using STM32 cards, the minimum cycle time that we have achieved is $10 \mathrm{~ms}$ as shown in Table I. we wanted to refine the configuration of the network by acting on the objects of the EPL in order to reach a cycle time less than $10 \mathrm{~ms}$. Unfortunately, under $10 \mathrm{~ms}$ the nodes neither interconnect nor exchange messages. This phenomenon refers to the integrated clock in each node ( $\mathrm{MN}$ or $\mathrm{CN})$ of the network.

\section{THE SAFETY OVER REAL-TIME ETHERNET}

The industrial communication in deterministic networks is far from being secure, it guarantees perfect synchronization among devices, and meets the temporal requirements (section
3) imposed by the deterministic kernel, and the standard but not all safety requirements. To ensure these requirements, we need to add safety measures at the top of the application layer. OpenSAFETY is such an application layer communication protocol. In Section 3, we discuss the requirement of minimum cycle time equal to $10 \mathrm{~ms}$. The implementation of openSAFETY protocol over EPL as shown in Fig. 7 duplicates the cycle time calculated in the section 3 . It will be $20 \mathrm{~ms}$. These results meet the temporal requirements of the safety standard for lift control system; Programmable Electronic components and Systems in Safety Related Applications for Lifts (PESSRAL). In fact, in case of danger, the PESSRAL requires an immediate and necessary stop of the lift car regardless its speed. After an outbreak, the maximum tolerated margin for the movement of the lift car is 1.2 meter [23]. Furthermore, we assume that the maximum speed of lift car is 2.5 meters per second, so the system will take:

$\frac{1.2}{2.5}=0.48$ second to react.

The mechanical actuators require 0.4 second to perform efficiently [23]. It remains to the communication systems to react within a maximum time of $80 \mathrm{~ms}$ (sensor, controller, actuators). The sensors require $30 \mathrm{~ms}$ to update their information. The actuators require $30 \mathrm{~ms}$ to process orders from the controller. With a cycle time equal to $20 \mathrm{~ms}$ we meet the requirements of the PESSRAL standard because: $20+30+30=80 \mathrm{~ms}$. This seems satisfactory for our project. OpenSAFETY allows creating communication systems requir-

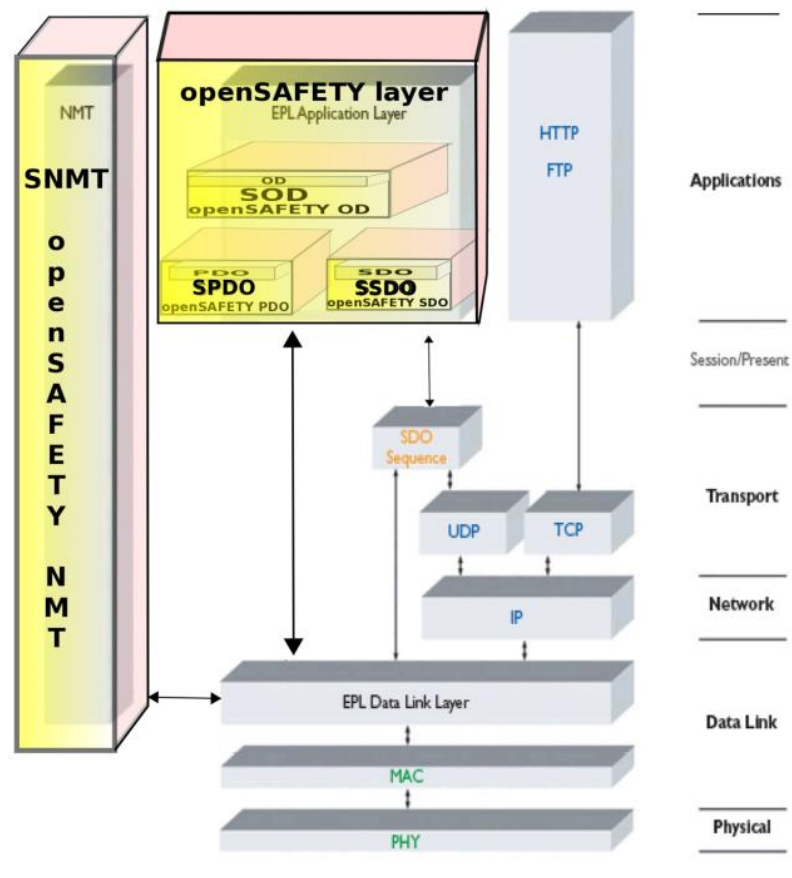

Fig. 7. OpenSAFETY over real-time Ethernet.

ing SIL 3 (Safety Integrity Level) according to PESSRAL 
[24]. It is a set of components offering services and security mechanisms for secure data exchange via networks unsecured [25]. For example:

- Time stamp: This timestamp mechanism allows associating with each frame the time and date of transmission in order to avoid duplication of frames.

- Time monitoring: This time monitoring can predict moments of frame arrival and thus can detect losses and delays.

- Identification: Each frame is identified by a unique identifier to prevent and detect any kind of integration.

- Cyclic Redundancy Check (CRC): To ensure the integrity of messages sent and to avoid the alteration and modification of data, OpenSAFETY uses the CRC.

- Frame format: Using different frame format allows the distinction between the standard frame and the Open SAFETY frames.

\section{CONCLUSION}

The innovative aspect of this collaborative project is the capacity to replace the classical safety chain composed of electromechanical components (serial electric contacts) by dedicated fieldbus networks. In this research project, we identified an adapted IP protocol to support dependability constraints for lift applications control. The mixed methodology allows integrating the communication architecture in the development in order to ensure the time performances based on deterministic operating system and safety by construction. Currently, the safety chain is not possible to diagnose. Indeed, our contribution will allow to perform a safety chain analysis. As a perspective, we can study the possibility to guarantee SIL3 in the worst-case scenario.The next task will be devoted to the modeling of a safe communication. This generic model will be included in the modeling of a deterministic core used in the ADN4SE project.

\section{ACKNOWLEDGMENT}

This research is supported by a grant of the BGLE-ADN4SE project (Atelier de Développement et Noyau pour (For) Systèmes Embarqués) supporting by the French industry ministry and lead by Sherpa and Krono Safe companies. The authors want to thanks the members of the workpackage lift for theirs analysis and specifications: Schneider, Sprinte, Itris automation.

\section{REFERENCES}

[1] B. Lamas, A. Soury, B. Saadallah, A. Lahmadi and O. Festor, "An Experimental Testbed and Methodology for Security Analysis of SCADA Systems", INRIA-Project-Team Madynes, Nancy, TR-443 (0249-0803), Dec. 2013.

[2] K. Stouffer, J. Falco and K. Scarfone, "Guide to industrial control systems (ICS) security", NIST special publication, 800-82, 2011.

[3] A. Soury, D. Genon-Catalot and J.M. Thiriet, "New lift safety architecture to meet PESSRAL requirements", World Symposium on Web Applications and Networking. WSWAN'2015. IEEE Conference, 2015.

[4] P. Neumann, "Communication in industrial automation?What is going on?", Control Engineering Practice, 2007, vol. 15, no 11, p. 13321347.

[5] G. Prytz, "A performance analysis of EtherCAT and PROFINET IRT", Emerging Technologies and Factory Automation. ETFA'08. IEEE Conference on. IEEE, 2008.
[6] ModBus, "The Modbus Organization", available on:www.modbus. org, 2015.

[7] O. Dolejs, P. Smolik, and Z. Hanzalek, "On the Ethernet use for realtime publish subscribe based applications", Factory Communication Systems, 2004. Proceedings. 2004 IEEE International Workshop on IEEE, 2004.

[8] Ethernet/IP, "Ethernet Industrial Protocol (EtherNet/IP)", available on:www.ethernet-ip.org, 2015.

[9] Real-Time Ethernet, "EtherNet/IP With Time Synchronization: Proposal for a Publicly Available Specification for Real-Time Ethernet”, Doc. IEC 65C/361/NP, 2004.

[10] Profinet, "PROFIBUS \& PROFINET International", available on:www.profibus.com, 2015.

[11] J. Feld, "PROFINET-scalable factory communication for all applications", Factory Communication Systems, 2004. Proceedings. 2004 IEEE International Workshop on. IEEE, 2004.

[12] Ethernet Powerlink Standardization Group, "Ethernet Powerlink, Communication Profile Specification version 1.2.0", available on:http://www.ethernet-powerlink.org, 2015.

[13] V. Kaczmarczyk, M. Sir and Z. Bradac, "Ethernet powerlink asynchronous phase examination", World Scientific and Engineering Academy and Society. WSEAS'05. international conference on Communications and information technology, 2011.

[14] EtherCAT, "EtherCAT Technology Group", available on:www.ethercat.org, 2015.

[15] E. Schemm, "SERCOS to link with ethernet for its third generation", Computing and Control Engineering 15.2 (2004): 30-33.

[16] D. Jansen and H. Buttner, "Real-time ethernet the EtherCAT solution", Computing and Control Engineering 15.1 (2004): 16-21.

[17] E. Lisova, E. Uhlemann, J. Akerberg and M. Bjorkman, 'Towards secure wireless TTEthernet for industrial process automation", Emerging Technologies and Factory Automation. ETFA 2014. IEEE Conference on. IEEE, 2014.

[18] P.G. Peón, H. kopetz and W. Steiner, "TTowards a reliable and high speed wireless complement to TTEthernet", Emerging Technologies and Factory Automation. ETFA 2014. IEEE Conference on. IEEE, 2014.

[19] W. Steiner, G. Bauer, B. Hall, and M. Paulitsch, "TTEthernet: TimeTriggered Ethernet", Time-Triggered Communication, R. Obermaisser, Ed. CRC Press, Aug 2011.

[20] G. Gena, L. Seno, A. Valenzano and S. Vitturi, "Performance analysis of Ethernet Powerlink networks for distributed control and automation systems", Computer Standards \& Interfaces, vol. 31, no 3, p. 566-572, 2009

[21] H. Xu, Y. Gao, K. Liu, B. Zhu and C. Zhang, "Research on Crosscommunication Based on Real-time Ethernet POWERLINK", Control and Decision Conference, The 26th Chinese CCDC, IEEE Conference, 2014.

[22] A. Soury, D. Genon-Catalot and J.M. Thiriet, "La sécurité des ascenseurs avec des communications Ethernet-Based Real-Time", Edito 2 Historique - Comités du JNCT 2014.

[23] PESSRAL:2008"A lift design and development of programmable elec tronic systems in safety-related applications for lifts (PESSRAL)", 3rd ed. ISO/FDIS22201:2008, 2008

[24] A. Elia, L. Ferrarini and C. Veber,, "Analysis of Ethernet-based safe automation networks according to IEC 61508", Emerging Technologies and Factory Automation. ETFA'06. IEEE Conference on. IEEE, 2006.

[25] "OpenSAFETY", version 1.4. June, 2014. 